\title{
Modeling magnetized star-planet interactions: boundary conditions effects
}

\author{
Antoine Strugarek ${ }^{1,2}$, Allan Sacha Brun ${ }^{2}$, Sean P. Matt ${ }^{3}$ and Victor \\ Reville $^{2}$ \\ ${ }^{1}$ Département de physique, Université de Montréal, \\ C.P. 6128 Succ. Centre-Ville, Montréal, QC H3C-3J7, Canada \\ email: strugarek@astro. umontreal.ca \\ ${ }^{2}$ Laboratoire AIM Paris-Saclay, CEA/Irfu Université Paris-Diderot CNRS/INSU, \\ F-91191 Gif-sur-Yvette \\ ${ }^{3}$ Department of Physics \& Astronomy, University of Exeter, Exeter EX2 4QL, UK
}

\begin{abstract}
We model the magnetized interaction between a star and a close-in planet (SPMIs), using global, magnetohydrodynamic numerical simulations. In this proceedings, we study the effects of the numerical boundary conditions at the stellar surface, where the stellar wind is driven, and in the planetary interior. We show that is it possible to design boundary conditions that are adequate to obtain physically realistic, steady-state solutions for cases with both magnetized and unmagnetized planets. This encourages further development of numerical studies, in order to better constrain and undersand SPMIs, as well as their effects on the star-planet rotational evolution.
\end{abstract}

Keywords. planet-star interactions; stars: winds, outflows; magnetohydrodynamics (MHD)

\section{Introduction}

The growing number of known exoplanet systems raises the question of how to properly define the habitability zone around a star (Kasting et al. 1993; Barnes et al. 2011). Its definition depends on the interactions existing between a planet and its host star, which are gravitational (tidal forces), magnetic (wind-planet interactions, hereafter referred to as SPMI) and radiative (e.g., stellar EUV ionisation flux). Magnetized interactions between a star and its orbiting planets have recently been suggested to be at the origin of a possibly enhanced planet detectability (Jardine \& Collier Cameron 2008; Fares et al. 2010; Miller et al. 2012). In the case of a close-in planet, these interactions may also be at the origin of anomalous stellar magnetic activity (Cuntz et al. 2000; Lanza 2008; Donati et al. 2008). It was also suggested that it could affect the star-planet rotational evolution (Laine et al. 2008; Pont 2009; Cohen et al. 2010; Vidotto et al. 2010; Lanza 2010). Theoretical work is needed to better understand SPMIs.

Based on a pioneering work done in the context of the satellites of Jupiter (Goldreich \& Lynden-Bell 1969; Kivelson et al. 2004), Laine \& Lin (2011) built an analytical model describing the various components of SPMIs in the case of unmagnetized planets. Pursuing the same goal, Lanza (2013) also developed semi-analytical models of SPMIs in the context of magnetized planets. However, a systematic numerical validation of those models still remains to be properly done (see Ip et al. 2004; Cohen et al. 2011, for first steps towards such a validation).

Focusing on close-in planets, the SPMIs include magnetic reconnection, magnetic field diffusion at the stellar surface and in the planet vicinity, radiation and ionisation processes in the planetary magnetosphere and magneto-sonic wave propagation. A numerical 
investigation of SPMI requires a careful description of those physical processes although it is generally not possible to treat all of them simultaneously with a unique model. Hence, specific strategies such as dedicated boundary conditions have to be developed to study SPMI from a global point of view. We detail in this work how to develop both stellar (section 2) and planetary (section 3) boundary conditions to globally model the different SPMI cases, within the MHD formalism.

\section{Stellar boundary conditions}

We model stellar winds following numerous previous analytical and numerical studies (Weber \& Davis 1967; Washimi \& Shibata 1993; Ustyugova et al. 1999; Keppens \& Goedbloed 2000; Matt \& Balick 2004; Matt et al. 2012). We use standard MHD theory to numerically model with the PLUTO code (Mignone et al. 2007) magnetized steady state flows anchored at the surface of a rotating star. We model winds driven by the thermal pressure of the stellar corona in a 2D axisymmetric cylindrical geometry (see Strugarek et al. 2012, for a more detailed description of the MHD model we use).

The steady-state wind solution can depend very sensitively on the type of boundary conditions that are imposed under the stellar surface. Because we want to use our model to study SPMIs, the stellar boundary conditions have to be able to both react and adapt to external stimuli originating from the orbiting planet. The design of a boundary

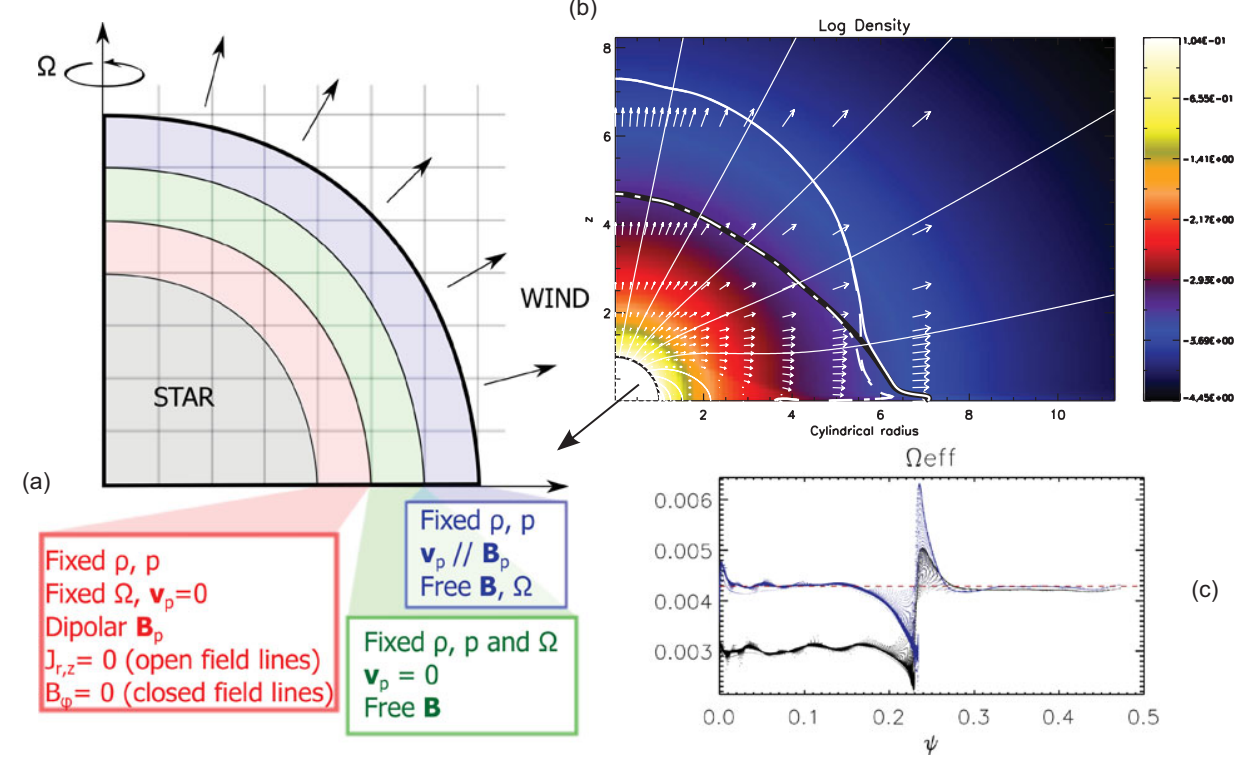

(b) Log Density

Figure 1. (a) Schematic of the multi-layer boundary condition ensuring good conservation properties of the MHD solution as well as reactivity to external stimuli. Fixed quantities are forced to the Parker wind solution. The subscript $p$ stands for the poloidal component $(\varpi, z)$ of vector in cylindrical coordinates. (b) Typical wind solution used for SPMI. The color map represents the logarithmic density, the white lines the poloidal magnetic field lines. The slow and fast Alfvèn surfaces are labeled by the dashed lines, and the arrows show velocity field. The stellar surface is labeled by a black quarter of a circle. The axes are in stellar radius units. (c) Effective rotation rate as a function of the streamfunction $\psi$ for good (blue dots) and bad (black dots) boundary conditions. The red dashed horizontal line labels the stellar rotation rate. Low values of $\psi$ correspond to open polar field lines and larger values of $\psi$ to closed equatorial field lines. Each dot corresponds to a grid point. 
condition satisfying those two conditions, and its associated stellar wind solution, are displayed in panels (a) and (b) of fig. 1.

We developed a layered boundary condition over which the stellar wind characteristics are progressively enforced as we go deeper under the stellar surface. This boundary condition ensures very good conservation properties (Lovelace et al. 1986; Zanni \& Ferreira 2009) along the magnetic field lines. This is exemplified in panel (c) of fig. 1. We display the effective rotation rate $\Omega_{\text {eff }} \equiv \frac{1}{\varpi}\left(v_{\phi}-\frac{v_{p}}{B_{p}} B_{\phi}\right)$ as a function of the streamfunction $\psi$ generating the poloidal magnetic field. In a steady-state, ideal MHD wind, $\Omega_{\text {eff }}$ should be constant along each field line and equal to $\Omega_{\star}$. The blue dots correspond to the boundary condition described in panel (a), and the black dots to a case where $B_{\phi}$ is set to 0 at all latitudes in the third boundary level. We observe that the target stellar rotation rate (dashed horizontal red line) is recovered only with the correct boundary conditions. Conservation errors exist at the open-closed field lines boundary $(\psi \sim 0.23)$, but they remain confined to very few grid points in the simulation domain. Finally, this boundary condition is intrinsically able to react to a perturbation by a planet orbiting a star by, e.g., modifying the stellar wind topology. We discuss now the importance of planetary boundary conditions when studying SPMIs.

\section{Planetary boundary conditions}

SPMIs are generally decomposed in two categories: the so-called unipolar and dipolar interactions (Zarka 2007), which refer to the cases of unmagnetized and magnetized planets. Both interactions can be modeled within the MHD formalism with an adequate boundary condition design. We detail in this section how to design such boundary conditions. The examples given here were all done for a planet with a radius of $r_{p}=0.1 r_{\star}$, a mass of $M_{p}=0.01 M_{\star}$, an orbital radius of $r_{\text {orb }}=3 r_{\star}$ and a resolution of $0.03 r_{p}$ at the planetary surface.
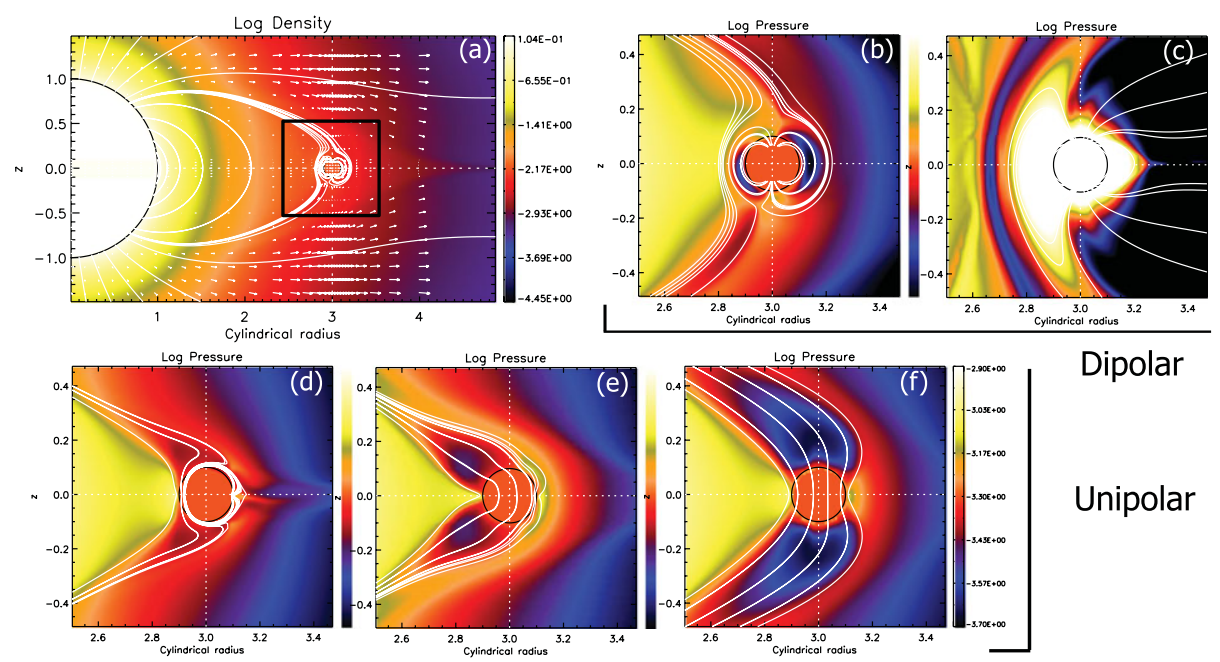

Dipolar

\section{Unipolar}

Figure 2. Zoom on planetary boundary conditions effects for dipolar (upper panels) and unipolar (lower panels) interactions. The color map represents the gas pressure in logarithmic scale, and the white lines the magnetic field lines. The planet surface is labeled by a black circle at 1 stellar radius. Panels (a) and (b) show the fiducial dipolar case, and panel (c) is the unrealistic case of a planet with a very high internal pressure. Panel (d) represents a Venus-like interaction and panels (e) and (f) two Io-Jupiter-like interactions. 
We consider the planet itself as a boundary condition. The PLUTO code allows one to define internal domains as boundary conditions over which all variables can be altered during the model evolution. In all cases, we set the poloidal velocity to zero and the azimuthal velocity to the keplerian velocity inside the planet. We also set the density and pressure values inside the planet to fiducial values which are consistent with its gravity field. These value have to be carefully prescribed since they can trigger undesirable effects in the vicinity of the planet. We give an example of a dipolar case in panels (a) and (b) of fig. 2 (the planetary magnetic field is simply enforced in the planetary interior in this case). A stable configuration is obtained when the magnetic pressure and the gas pressure equilibrate at the interface between the planetary magnetosphere and the stellar wind. The ram pressure plays little role here because the planet we consider is in the so called dead-zone of the stellar wind, in which the poloidal velocity is negligible. We show in panel (c) the exact same simulation for an extreme case where we multiplied the internal pressure of the planet by a factor of 20 . The former pressure balance then fails and a wind is driven from the planet itself. The planetary dipole opens up and a shock eventually creates at the interface between the two "winds". Such undesirable effects may also be obtained by varying the density of the interior of the planet. Hence, any SPMI model must be developed to minimize such undesirable effects in the final solution.

Modeling a planet in the unipolar case is a bit more complex than in the dipolar case. Two classes of unipolar interactions can indeed be distinguished: Venus-like interaction (case V) and Io-Jupiter like interaction (case IJ). Note however that in both cases, we consider a planet located inside the stellar wind dead-zone, at $r_{p}=3 R_{\star}$.

In case $\mathrm{V}$, the ionisation of the planetary atmosphere by the stellar EUV radiation flux allows the creation of a ionosphere which acts as a barrier between the stellar wind magnetic field and the unmagnetized interior of the planet (Russell 1993). Depending on the stellar wind conditions around the planet, an induced magnetosphere may then be sustained on secular time scales. We show case $\mathrm{V}$ in panel (d) of fig. 2. The ionosphere is modeled as a very thin $\left(<0.2 r_{p}\right)$ highly conductive boundary layer under the planetary surface. The wrapping of the magnetic field lines around planet (Russell 1993) is naturally recovered.

In case IJ, no ionosphere is created and the stellar wind magnetic field pervades inside the planet. The SPMI then depends on the ratio of electrical conductivities between the planetary interior and the stellar surface where the magnetic field lines are a priori anchored. This ratio sets the effective drag the planet is able to induce on the stellar wind magnetic field lines. We use the ability of the PLUTO code to add extra ohmic diffusion in the planet interior to model it and show in figure 2 two extreme cases in which magnetic field lines are dragged (panel e) or diffused (panel f) by the planet. In all cases, we obtain a statistical steady state in which the SPMI can be analyzed in details.

\section{Conclusions}

We showed in this work that is it possible to model the global, magnetized and nonlinear interactions between a star and a planet, within the MHD formalism. It requires a careful development of adequate boundary conditions to represent the various interaction cases. We showed that boundary conditions play a very important role both at the stellar surface and in the planetary interior. Steady state solutions could be found in the dipolar case as well as in both the Venus-like and Io-Jupiter-like unipolar cases.

The SPMI model we developed will be useful for exploring stable interaction configurations between a close-in planet and its host star. In addition, it will enable quantitative predictions of rotational evolution of star-planet systems due to the effective magnetic 
torques which develop in the context of dipolar and unipolar interactions (Strugarek et al., in prep). Finally, such models could also be used to study potential SPMI induced emissions, which we will analyze in a future work.

\section{Acknowledgements}

We thank A. Mignone and his team for making the PLUTO code open-source. We thank A. Cumming, R. Pinto, C. Zanni and P. Zarka for inspiring discussions on starplanet magnetized interactions. This work was supported by the ANR TOUPIES and the ERC project STARS2. We acknowledge access to supercomputers through GENCI project 1623 and Prace infrastructures. A. Strugarek acknowledges support from the Canada's Natural Sciences and Engineering Research Council.

\section{References}

Barnes, R., Meadows, V. S., Domagal-Goldman, S. D., et al. 2011, 16th Cambridge Workshop on Cool Stars, 448, 391

Cohen, O., Drake, J. J., Kashyap, V. L., Sokolov, I. V., \& Gombosi, T. I. 2010, ApJ, 723, L64

Cohen, O., Kashyap, V. L., Drake, J. J., et al. 2011, ApJ, 733, 67

Cuntz, M., Saar, S. H., \& Musielak, Z. E. 2000, ApJ, 533, L151

Donati, J.-F., Moutou, C., Fares, R., et al. 2008, MNRAS, 385, 1179

Fares, R., Donati, J.-F., Moutou, C., et al. 2010, MNRAS, 406, 409

Goldreich, P. \& Lynden-Bell, D. 1969, ApJ, 156, 59

Ip, W.-H., Kopp, A., \& Hu, J.-H. 2004, ApJ, 602, L53

Jardine, M. \& Collier Cameron, A. 2008, A\& A, 490, 843

Kasting, J. F., Whitmire, D. P., \& Reynolds, R. T. 1993, Icarus, 101, 108

Keppens, R. \& Goedbloed, J. P. 2000, ApJ, 530, 1036

Kivelson, M. G., Bagenal, F., Kurth, W. S., et al. 2004, In: Jupiter. The planet, 513

Laine, R. O. \& Lin, D. N. C. 2011, ApJ, 745, 2

Laine, R. O., Lin, D. N. C., \& Dong, S. 2008, ApJ, 685, 521

Lanza, A. F. 2008, $A \mathscr{E} A, 487,1163$

-. 2010, $A \& A, 512,77$

-. 2013, A\& $A, 557,31$

Lovelace, R. V. E., Mehanian, C., Mobarry, C. M., \& Sulkanen, M. E. 1986, ApJS, 62, 1

Matt, S. \& Balick, B. 2004, ApJ, 615, 921

Matt, S. P., MacGregor, K. B., Pinsonneault, M. H., \& Greene, T. P. 2012, ApJL, 754, L26

Mignone, A., Bodo, G., Massaglia, S., et al. 2007, ApJS, 170, 228

Miller, B. P., Gallo, E., Wright, J. T., \& Dupree, A. K. 2012, ApJ, 754, 137

Pont, F. 2009, MNRAS, 396, 1789

Russell, C. T. 1993, Reports on Progress in Physics, 56, 687

Strugarek, A., Brun, A. S., Matt, S. P., \& Reville, V., in preparation

Strugarek, A., Brun, A. S., \& Matt, S. 2012, in SF2A-2012: Proceedings of the Annual meeting of the French Society of Astronomy and Astrophysics. Eds.: S. Boissier, 419-423

Ustyugova, G. V., Koldoba, A. V., Romanova, M. M., Chechetkin, V. M., \& Lovelace, R. V. E. 1999, ApJ, 516, 221

Vidotto, A. A., Opher, M., Jatenco-Pereira, V., \& Gombosi, T. I. 2010, ApJ, 720, 1262

Washimi, H. \& Shibata, S. 1993, MNRAS, 262, 936

Weber, E. J. \& Davis, L. J. 1967, ApJS, 148, 217

Zanni, C. \& Ferreira, J. 2009, A\&GA, 508, 1117

Zarka, P. 2007, Planetary and Space Science, 55, 598 